\title{
Correlation of NUCB2/Nesfatin-I with Cytokine Levels in Primary Open-Angle Glaucoma
}

\author{
Milena Pahlitzsch ${ }^{1}$ \\ Raphaela Fritsche- \\ Guenther ${ }^{2}$ \\ Inga Pompös (D) \\ Dominika Pohlmann' \\ Anna-Karina B Maier' \\ Sibylle Winterhalter' \\ Carl Erb ${ }^{3}$ \\ Anne Rübsam' \\ 'Charité - Universitätsmedizin Berlin, \\ Corporate Member of Freie Universität \\ Berlin, Humboldt-Universität zu Berlin, \\ and Berlin Institute of Health, Berlin, \\ Germany; ${ }^{2}$ Berlin Institute of Health at \\ Charité - Universitätsmedizin Berlin, \\ Metabolomics Platform, Berlin, Germany; \\ ${ }^{3}$ Eye clinic Wittenbergplatz, Berlin, \\ Germany
}

Correspondence: Milena Pahlitzsch Charité - Universitätsmedizin Berlin, Corporate Member of Freie Universität Berlin, Humboldt-Universität zu Berlin, and Berlin Institute of Health, Berlin, Germany

Tel +4930450654326

$\mathrm{Fax}+493084454450$

Email milena.pahlitzsch@charite.de
Purpose: Nesfatin-1 is produced in various tissues of the body including the hypothalamus. Neuroprotective properties of the neuropeptide hormone Nesfatin-1 were recently described. The aim of the study was to analyze the molecule Nesfatin-1 as a possible biomarker in POAG with neuroprotective properties pointing out the retinal-hypothalamic axis as target site in POAG and to obtain a molecular signature of cytokines in POAG as neuroinflammatory processes are a key factor of glaucoma development.

Methods: In this study, $\mathrm{n}=35$ patients with moderate and advanced POAG (mean age $65.0 \mathrm{y}$, IOP $13.9 \pm 3.0 \mathrm{mmHg}$ ) and $\mathrm{n}=35$ healthy controls (mean age $51.6 \mathrm{y}$, IOP $14.3 \pm 2.7 \mathrm{mmHg}$ ) were included. Clinical parameters including IOP, cup to disc ratio (CDR), glaucoma medication and retinal nerve fiber layer thickness (RNFL) were recorded. Plasma was collected for NUCB2/nesfatin-1 measurement using a Nesfatin-1 ELISA and for detection of 13 inflammatory cytokines using a multiplex bead-based immunoassay (MagPix). Multiple linear regression analysis was performed to adjust for confounding factors.

Results: Sex-independent or sex-dependent variables showed no significant differences in the Nesfatin-1 level ( $p>0.05)$. As a trend, an increase in NUCB2/nesfatin-1 in male glaucoma patients was found. Increased concentrations of 11 cytokines (GM-CSF, Interferon- $\gamma$, Interleukin-1 $\beta$, IL-2, 4, 5, 6, 7, 10, 12 and TNF- $\alpha$ ) were detected in POAG. The female glaucoma patients demonstrated elevated cytokine concentrations compared to male patients. NUCB2/nesfatin-1 showed a significant correlation to IL-2 and IL-13 levels in POAG. Stepwise multiple regression analysis showed no difference in NUCB2/nesfatin-1 level between POAG and healthy controls after adjusting for sex and age (all $\mathrm{p}>0.05$ ).

Conclusion: As a trend, male POAG patients showed increased plasma NUCB2/nesfatin-1 levels. We further found inflammation as contributing factor to the pathogenesis of glaucoma, with a greater inflammatory response in women.

Keywords: NUCB2/nesfatin-1, primary open-angle glaucoma, molecular signature, cytokines

\section{Introduction}

Glaucoma is a complex disorder characterized by the loss of retinal ganglion cells (RGCs) and their axons, damage to the optic nerve, and gradual loss of the visual field. It represents the second leading cause of irreversible blindness worldwide - accounting for $8 \%$ of global blindness as reported by the World Health Organization. ${ }^{1}$ Glaucoma is subdivided into two main groups: Open-angle glaucoma (OAG) and closed-angle glaucoma. Open-angle glaucoma can be classified depending on the intraocular pressure (IOP) into high-pressure glaucoma (primary open angle glaucoma, POAG) and low-pressure glaucoma (normal tension glaucoma, NTG). 
During the past century up to date, we still face unsatisfying therapeutic regimens where an elevated IOP is the only treatable risk factor in glaucoma. However, a subset of patients present an IOP within the normal range and still develop glaucoma (NTG). It appears that progressive RGC loss might not be directly correlated to the extent of the IOP level in all patients. Analyses of the transcriptome and proteome of glaucomatous eyes have shown that complex molecular events are associated with the development of glaucoma. ${ }^{2,3}$ Thus, both in glaucoma patients and in rodent models of glaucoma, there is accumulating evidence for a potential role of hormones and immunological processes in glaucomatous optic neuropathy. ${ }^{4}$

NUCB2/nesfatin-1 is known to be expressed throughout the body, including fat tissue, pancreatic beta cells, gastric mucosa and the hypothalamus of mammals (Figure 1). ${ }^{5,6}$ Hypothalamic nucleobindin 2 (NUCB2) is the precursor of Nesfatin-1. NUCB2/nesfatin-1 is able to cross the bloodbrain barrier in any direction by nonsaturable mechanisms. ${ }^{7-9}$ NUCB2/nesfatin-1 has been detected in different regions of the rat hypothalamus, especially in the supraoptic nucleus, paraventricular nucleus, lateral hypothalamic area, and arcuate nucleus. ${ }^{5,6}$ Pointing out the arcuate nucleus, Gong et al finds that this structure and its gammaAminobutyric acid (GABA) receptors are involved in the regulation of intraocular pressure. ${ }^{6}$ GABA receptor antagonists lead to an IOP reduction showing a decline of retinal ganglion cell apoptosis $(\mathrm{p}<0.01) .{ }^{6}$ An increase of IOP $(+7.1 \pm$ $1.9 \mathrm{mmHg}$ ), intracerebral pressure, and the translaminar pressure gradient was seen after chemically stimulated neurons of a rodent hypothalamus. ${ }^{10}$ Circadian fluctuations in IOP and the translaminar pressure gradient may be modulated by the hypothalamus. ${ }^{10,11}$

Additionally, comparative research is linked to NUCB2/ nesfatin-1 as a regulator molecule of stress responses, especially under inflammatory conditions. ${ }^{7,12}$ NUCB2/nesfatin-1 might show this effect by activating NUCB2 secreting parvocellular neurons. ${ }^{7}$ As a multifactorial disease there is evidence that glaucomatous progression is due to pro-inflammatory responses. $^{12-17}$

In a recent study, Özsavci et al found a neuroprotective effect of NUCB2/nesfatin-1 in a cerebral trauma model, especially anti-apoptotic and anti-inflammatory properties with a significant decrease of immune mediators (tumor necrosis factor- $\alpha$, interleukin- $1 \beta$, interleukin- 6 , lipid peroxidation, protein carbonylation, caspase- 3 activity and myeloperoxidase activity). ${ }^{12}$ Neuroinflammation is consequently a key process in glaucoma. ${ }^{18-20}$ Evidence so far has demonstrated altered expression levels of certain inflammatory cytokines in the aqueous humour (AH) and peripheral blood of glaucomatous eyes of POAG and closed-angle glaucoma patients. These include the following factors in the serum: soluble Interleukin-2 receptor (sIL-2R), IL-2, IL-4, IL-6, IL-10, IL-23 and tumor necrosis factor- $\alpha$ (TNF$\alpha) .{ }^{21,22}$ Additionally, the following factors have been found to be elevated in the aqueous humour: transforming growth factor beta-1 (TGF- $\beta 1$ ), TGF- $\beta 2$, IL-1 $\alpha$, IL-2, IL-5, IL-6, IL8, IL-9, IL-10, IL-12, IL-23, $\alpha$-serum amyloid A, interferon- $\gamma$ (IFN- $\gamma$ ), and chemokine (C-X-C motif) ligand (CXL) 9. ${ }^{3,21-}$ ${ }^{24}$ Furthermore, numerous pathways - known to be critical regulators of neuroinflammation-show gene-expression differences in glaucoma. ${ }^{25,26}$ In glaucoma, it may be an elevated IOP, not necessarily a foreign antigen, that triggers an innate immune response, which usually involves resident immune cells, such as microglia, as well as the infiltration of macrophages/monocytes. ${ }^{27}$ Recent studies have provided evidence indicating a link between elevated IOP and T cellmediated autoimmune responses, which might play an important role in the pathogenesis of glaucomatous neurodegeneration. ${ }^{28}$ Reactive glial responses and lowgrade inflammation may initially represent an adaptive reaction of the retina to primary stress stimuli; whereas sustained and excessive glial reactions lead to expanded immune responses, including adaptive immunity, that contribute to progressive neural damage in glaucoma. In light of animal studies that are beginning to show that modulation of neuroinflammation may be a promising therapeutic strategy, a renewed effort to study the inflammatory response in human glaucoma is warranted. ${ }^{28-31}$

NUCB2/nesfatin-1 may be a marker with neuroprotective properties for the inflammatory situation in POAG having an impact on the retino-hypothalamic tract (Figure 1).

The purpose of this study was to analyze NUCB2/ nesfatin- 1 and a molecular signature of inflammatory cytokines in the plasma of POAG patients as biomarkers for the glaucomatous neuroinflammation.

\section{Methods}

\section{Patients and Sample Collection}

This study was designed as a prospective observational study and included 70 patients $(n=35$ POAG patients and $n=35$ healthy controls) seen at the Glaucoma Department of the Charité University clinic Berlin between February 2019 and March 2020. The Ethics Committee of the Charité University Berlin approved the study protocol (EA4/080/19), and the 


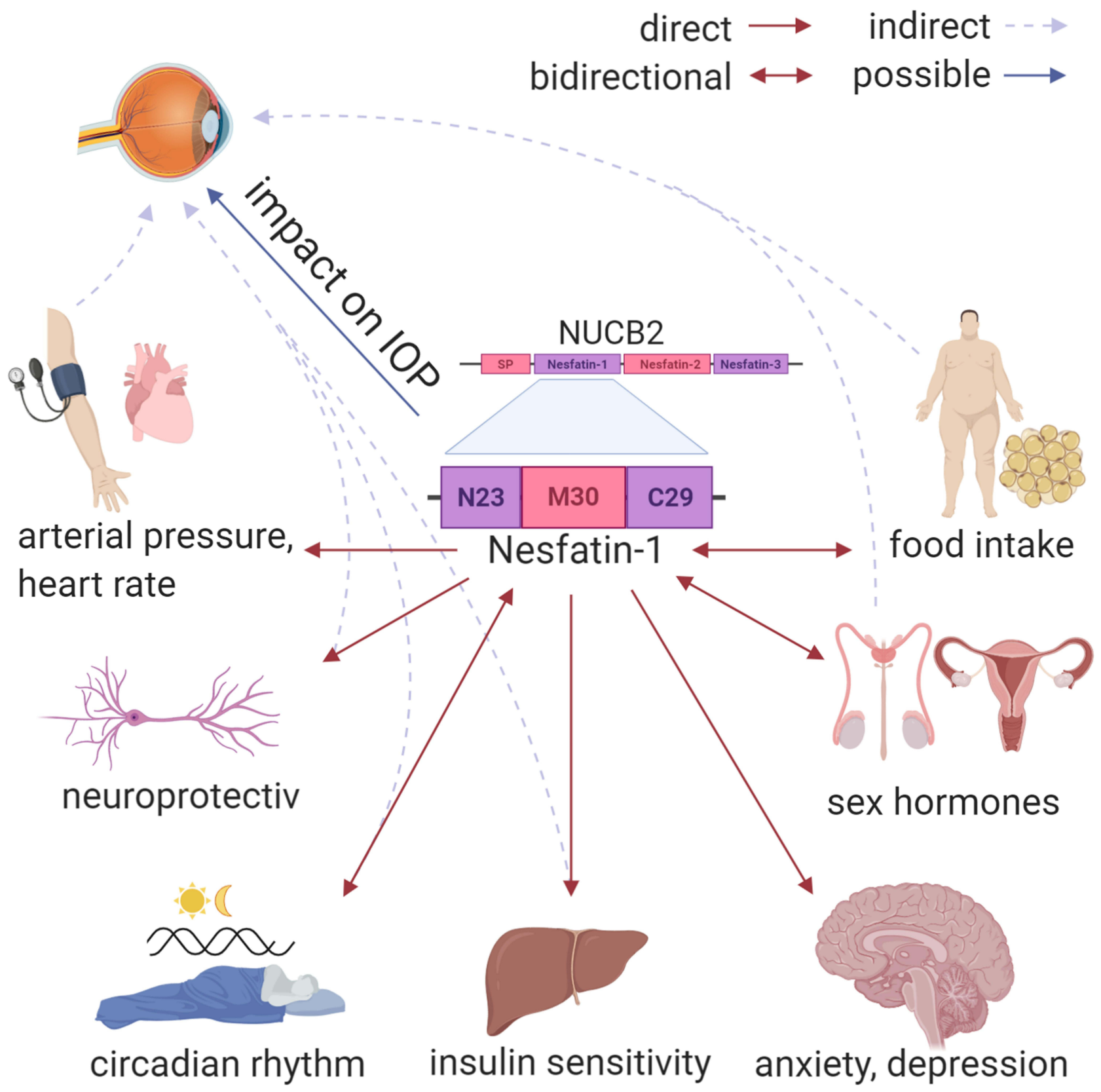

Figure I The impact of Nesfatin-I on the hypothalamus. Red arrows show a direct effect on different central and peripheral organs and processes. Grey arrows with a dashed line show an indirect impact of Nesfatin-I, via the impact on peripheral processes which has an influence as a glaucoma risk factor. The blue arrow shows the possible direct impact of Nesfatin-I on the intraocular pressure via the retino-hypothalamic axis.

procedures applied conformed to the tenets of the Declaration of Helsinki. Informed consent was obtained from all of the participants.

Open-angle glaucoma was diagnosed by the presence of an open iridocorneal angle, glaucomatous cupping of the optic disc and visual field defects (Humphrey Visual Field Analyzer, Carl Zeiss, Dublin, CA) without signs of secondary glaucoma. The IOP was measured by Goldmann applanation tonometry (mmHg). The inclusion criteria consisted of patients with
POAG with moderate or advanced glaucoma and with a cup to disc ratio (CDR) of 0.6 or higher (clinically and Heidelberg retina tomography HRT based, HRT III, Heidelberg Engineering, DE). The visual field parameters 'mean deviation (MD) and pattern standard deviation (PSD)' were obtained and included patients with $\mathrm{MD}>6 \mathrm{~dB}$ and PSD $>4 \mathrm{~dB}$. Exclusion criteria were patients who had received any intraocular surgery within 6 months prior to the beginning this study or patients with any signs of secondary glaucoma. Individuals with 
systemic inflammatory, metabolic or autoimmune diseasessuch as diabetes and hypertensive blood pressure - and any pre-existing ocular disease other than cataract were excluded from the study. The control group was composed of healthy individuals without any evidence of glaucomatous optic neuropathy or conferring ocular disease.

Clinical data were collected including IOP, glaucoma medication, glaucoma parameters (cup to disc ratio (CDR), funduscopy) and the retinal nerve fiber layer thickness (RNFL) based on the optical coherence tomography (OCT Zeiss, DE).

Plasma samples were collected in all subjects at one designated point in time between 7.00 am and 9.00 am as a circadian rhythm of the NUCB2/nesfatin-1 level in the plasma was described in the literature. ${ }^{32}$ Plasma NUCB2/ nesfatin-1 levels can vary by body weight or BMI; thus, these informations of the participants were also collected.

For the NUCB2/nesfatin-1 measurement, plasma from patients with POAG and from healthy donors were collected and analyzed using the Nesfatin-1 ELISA from Cusabio (CSB-E15050h). A TECAN plate reader was used to measure the optical density at $450 \mathrm{~nm}$ with correction wavelength of $40 \mathrm{~nm}$. For the inflammatory cytokine determination, the plasma was analyzed using a multiplex bead-based immunoassay technique ( $\mathrm{T}$ Cell Panel Premixed 13-plex, Merck) measured at the MagPix system (Merck) according to the manufacturer's instructions. Each compound in each sample is in minimum 30 times measured (30 beads were counted) and a median mean fluorescent intensity is given by the software. We have assessed the levels of granulocyte macrophage-colony stimulating factor (GM-CSF), IFN- $\gamma$, interleukin (IL)-1 $\beta, 2,4,5,6,7$, 8, 10, 12 and 13 and TNF- $\alpha$. Milliplex Analyst software was used for data analysis.

\section{Statistics}

Statistical analysis was performed using SPSS v.20.0 (SPSS Inc. Chicago, IL, USA). For calculating the sample size, the measurement of Nesfatin-1 in plasma is defined as the primary target variable. When examining a presumed continuous criterion with a significance level of $\alpha=5 \%$ and a power of $(1-\beta)=95 \%$ - and that also has a discernable difference of at least $85 \%$ of the standard deviation in independent samples - a result of the necessary minimum case numbers of 37 patients per group is reached. Due to the predesigned multiplex assay sample size by the manufacturer, only 35 patients could be analyzed.
Categorical variables were shown as frequencies and proportions and analyzed using $\chi^{2}$ test. The normality of the distribution of numeric variables was examined using the Kolmogorov-Smirnov test. The differences between groups at baseline were determined via unpaired $t$-test. The correlation between NUCB2/nesfatin-1 or cytokine concentration and clinical data was determined by Spearman's rank-order correlation test. Stepwise multiple linear regression analysis was performed to estimate the linear relationship of NUCB2-Nesfatin-1 as the main dependent variable in healthy patients and patients with glaucoma as independent variable including possible confounders as age and sex. $\mathrm{P}$ values less than 0.05 were considered as statistically significant. From the concentrations of all biological replicates, the ratio for a variety of tested conditions was calculated. For better visualization, the $\log 2$ ratio was generated. $P$ values were calculated using unpaired Student's $t$-test, with an adjusted $\mathrm{p}<0.05$ as the cut-off for significance.

\section{Results}

Seventy patients were included in the study: 35 eyes with POAG (mean age $65.0 \pm 8.9$, male $63.8 \pm 8.3$, female $64.1 \pm$ 10.8 ) and 35 healthy controls (mean age $51.6 \pm 17.7$, male $47.3 \pm 18.9$, female $55.1 \pm 16.3$ ). The sex-dependent distribution was 18 male and 17 female glaucoma patients and 13 male and 22 female control patients. The IOP was $13.9 \pm$ $3.0 \mathrm{mmHg}$ for the POAG eyes and $14.3 \pm 2.7 \mathrm{mmHg}$ in the control group. The complete demographic data and other clinical characteristics are summarized in Table 1.

In the current study, no significant sex-independent or sex-dependent differences in the NUCB2/nesfatin-1 level could be shown ( $>0.05$, Figure 2$)$. As a trend, an increase of NUCB2/nesfatin-1 in male glaucoma patients was found compared to the control group.

NUCB2/nesfatin-1 showed a significant correlation to IL-2 and IL-13 cytokine levels in the glaucoma cohort, but not in the healthy control. No significant difference could be detected between NUCB2/nesfatin-1 and the clinical parameters in both cohorts (Table 2).

To screen for markers of POAG, we determined the levels of 13 cytokines in the peripheral blood. Increased concentrations of 11 cytokines (GM-CSF, IFN- $\gamma$, IL-1 $\beta, 2$, $4,5,6,7,10,12$ and TNF- $\alpha$ with $7 / 11$ statistical significance) were sex-independently present in glaucoma patients in comparison to the control group - except of IL13 and IL-8 (Table 3, Figures 3 and 4). 
Table I Descriptive statistics of the Study Cohort

\begin{tabular}{|l|l|l|l|}
\hline Characteristics & POAG & Control & $P$-value \\
\hline Total no. of eyes & 35 & 35 & \\
Age, mean (SD), year & $65.0(8.9)$ & $51.6(17.7)$ & 0.001 \\
Sex, no. of M/F & $18 / 17$ & $13 / 22$ & 0.28 I \\
Body weight, kilogram & 70.0 & 74.5 & 0.058 \\
BMI & 23.6 & 24.1 & 0.689 \\
IOP OD, mean (SD), mmHg & $13.9(3.0)$ & $14.3(2.7)$ & 0.608 \\
CDR OD, mean (SD) & $0.8(0.2)$ & $0.3(0.05)$ & $<0.001$ \\
No. of glaucoma medications & $1.9(1.2)$ & - & \\
RNFL OD, mean (SD), $\mu \mathrm{m}$ & $78.6(20.0)$ & $96.7(8.9)$ & 0.002 \\
Mean defect visual field OD, diopter & 9.4 & 1.2 & $<0.00 \mathrm{I}$ \\
Pattern standard deviation OD, diopter & 4.1 & 0.9 & $<0.00 \mathrm{I}$ \\
\hline
\end{tabular}

Notes: mean \pm standard deviation, T: $\mathrm{p}$-value of Student's $t$-test; $\chi^{2}$ : chi squared value of chi squared test.

Abbreviations: IOP, intraocular pressure; CDR, cup to disc ratio; RNFL, retinal nerve fiber layer.

Comparing glaucoma patients to the control group, the female patients showed higher cytokine concentrations (except of IL-13 and IL-8), while the male patients showed a mixed pattern of up- and downregulation. In detail the values in $\mathrm{pg} / \mathrm{mL}$ for female glaucoma patients versus female control patients are GM-CSF: 27.4 versus $18.9, \mathrm{p}=0.022$; IFN- $\gamma$ : 12.3 versus $8.2, p<0.001$; IL-1 $\beta: 2.18$ versus 1.27 , $\mathrm{p}<0.001$; IL-2: 3.5 versus $2.42, \mathrm{p}=0.007$; IL-4: 40.4 versus 26.2, $\mathrm{p}=0.016$; IL-5: 7.1 versus 5.0, $\mathrm{p}=0.008$; IL-6: 2.7 versus 1.6, $\mathrm{p}<0.001$; IL-7: 13.9 versus $10.8, \mathrm{p}=0.001$; IL-8: 4.2 versus $4.8, \mathrm{p}=0.490$; IL-10: 19.7 versus $12.1, \mathrm{p}=0.005$; IL12: 3.3 versus $2.2, \mathrm{p}<0.001$, IL-13: 5.2 versus $5.4, \mathrm{p}=0.782$, TNF- $\alpha$ : 2.9 versus $2.4, \mathrm{p}=0.115$.

In addition, the values in $\mathrm{pg} / \mathrm{mL}$ for male glaucoma patients versus male control patients are GM-CSF: 22.8 versus $20.4, \mathrm{p}=0.485$; IFN- $\gamma$ : 10.4 versus $7.3, \mathrm{p}=0.015$; IL$1 \beta$ : 1.9 versus $1.6, \mathrm{p}=0.343$; IL-2: 2.8 versus $2.9, \mathrm{p}=0.915$; IL-4: 33.1 versus 25.1, $\mathrm{p}=0.182$; IL-5: 5.9 versus 5.4, $\mathrm{p}=0.598$; IL-6: 2.1 versus $1.7, \mathrm{p}=0.273$; IL-7: 11.7 versus 12.4, $\mathrm{p}=0.492$; IL-8: 3.9 versus $6.4, \mathrm{p}=0.139$; IL-10: 18.4 versus $12.4, \mathrm{p}=0.027$; IL-12: 3.1 versus $3.0, \mathrm{p}=0.952$, IL-13: 3.6 versus $3.9, \mathrm{p}=0.618$. TNF- $\alpha$ : 2.8 versus $2.8, \mathrm{p}=0.935$

In the glaucoma group, female patients showed increased cytokine concentrations for all upregulated cytokines except for TNF- $\alpha$ compared to male patients (Figure 4). In detail the values in $\mathrm{pg} / \mathrm{mL}$ for female versus male patients are GM-CSF: 27.4 versus $22.8, \mathrm{p}=0.245$; IFN- $\gamma$ : 12.3 versus $10.3, \mathrm{p}=0.144$; IL-1 $\beta$ : 2.18 versus $1.92, p=0.326$; IL-2: 3.5 versus 2.8 , $\mathrm{p}=0.109$; IL-4: 40.4 versus $33.1, \mathrm{p}=0.181$; IL-5: 7.1 versus $5.9, \mathrm{p}=0.157$; IL-6: 2.7 versus $2.1, \mathrm{p}=0.091$; IL-7: 13.9 versus

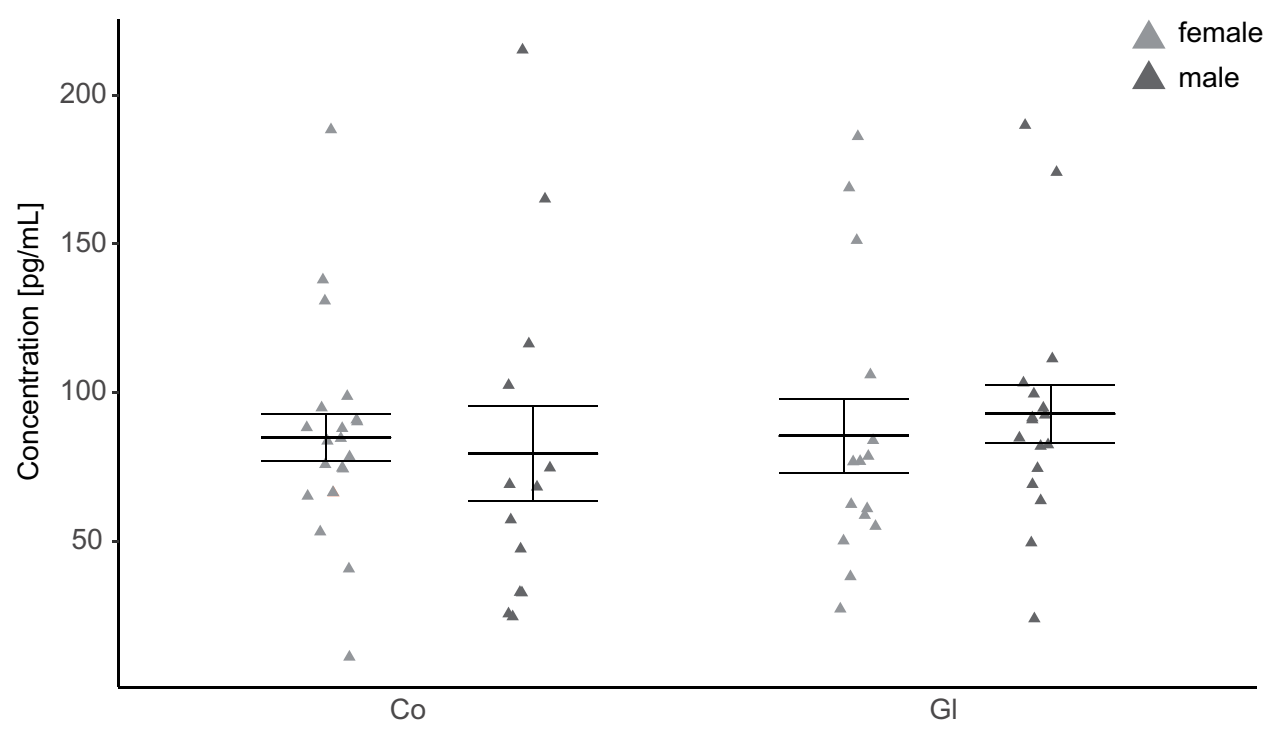

Figure 2 Jitter plot analysis of the measured NUCB2/nesfatin-I concentrations in primary open-angle glaucoma (GI) and healthy controls (Co). 
Table 2 Correlation Between NUCB2/Nesfatin-I Concentration and Clinical Parameters (IOP, CDR, RNFL, Number of Glaucoma Medication) and Cytokine Levels of Primary Open-Angle Glaucoma (POAG) and Control Patients

\begin{tabular}{|l|c|c|c|c|}
\hline \multirow{2}{*}{ Analytes } & \multicolumn{2}{|c|}{ Control } & \multicolumn{2}{c|}{ Glaucoma } \\
\cline { 2 - 5 } & $\rho$ & p-value & $\rho$ & p-value \\
\hline IOP OD $(\mathrm{mmHg})$ & -0.170 & 0.359 & 0.152 & 0.415 \\
CDR OD & -0.097 & 0.586 & 0.175 & 0.346 \\
RNFL OD $(\mu \mathrm{m})$ & 0.059 & 0.760 & -0.412 & 0.080 \\
Glaucoma medication & - & - & 0.213 & 0.250 \\
GM-CSF & 0.287 & 0.100 & 0.157 \\
IFN y & 0.016 & 0.929 & -0.26 I & 0.210 \\
IL-I0 & 0.173 & 0.327 & -0.23 I & 0.894 \\
IL-I2 & 0.088 & 0.622 & 0.025 & 0.148 \\
IL-I3 & -0.013 & 0.947 & -0.266 & 0.044 \\
IL-Ib & -0.005 & 0.977 & -0.364 & 0.104 \\
IL-2 & 0.188 & 0.286 & -0.302 & 0.018 \\
IL-4 & -0.076 & 0.673 & -0.147 & 0.43 . \\
IL-5 & 0.173 & 0.327 & -0.192 & 0.300 \\
IL-6 & -0.073 & 0.702 & -0.25 I & 0.173 \\
IL-7 & 0.234 & 0.184 & -0.068 & 0.717 \\
IL-8 & 0.155 & 0.414 & -0.087 & 0.646 \\
TNFa & 0.305 & 0.080 & 0.053 & 0.775 \\
\hline
\end{tabular}

Abbreviations: IOP, intraocular pressure; CDR, cup to disc ratio; RNFL, retinal nerve fiber layer; GM-CSF, granulocyte macrophage-colony stimulating factor; IFN, interferon; IL, interleukin; $\rho$, Spearman correlation coefficient.

Table 3 Map of the Log2 of the Mean Female/Male or Glaucoma/Control Ratios of the Analyte Concentration (Pg/mL)

\begin{tabular}{|c|c|c|c|c|c|}
\hline \multirow[t]{2}{*}{ Analytes } & Female+Male & Female/Male & Female/Male & Female & Male \\
\hline & Glaucoma/Control & Glaucoma & Control & \multicolumn{2}{|c|}{ Glaucoma/Control } \\
\hline GM-CSF & 0.366 & 0.261 & -0.109 & 0.535 & 0.165 \\
\hline $\mathrm{IFN}-\gamma$ & 0.518 & 0.247 & 0.170 & 0.579 & 0.501 \\
\hline IL-10 & 0.635 & 0.104 & -0.025 & 0.696 & 0.567 \\
\hline IL-12 & 0.342 & 0.099 & -0.456 & 0.578 & 0.024 \\
\hline IL-13 & -0.153 & 0.501 & 0.478 & -0.070 & -0.093 \\
\hline IL-I $\beta$ & 0.546 & 0.180 & -0.340 & 0.769 & 0.249 \\
\hline IL-2 & 0.289 & 0.325 & -0.259 & 0.548 & -0.035 \\
\hline IL-4 & 0.500 & 0.286 & 0.064 & 0.618 & 0.395 \\
\hline IL-5 & 0.333 & 0.270 & -0.110 & 0.507 & 0.127 \\
\hline IL-6 & 0.512 & 0.351 & -0.080 & 0.711 & 0.280 \\
\hline IL-7 & 0.162 & 0.250 & -0.203 & 0.365 & -0.089 \\
\hline IL-8 & -0.422 & 0.111 & -0.410 & -0.200 & -0.721 \\
\hline $\mathrm{TNF} \alpha$ & 0.143 & 0.056 & $-0.17 \mid$ & 0.238 & 0.011 \\
\hline
\end{tabular}

11.7, $\mathrm{p}=0.060$; IL-8: 4.2 versus $3.9, \mathrm{p}=0.540 ; \mathrm{IL}-10: 19.7$ versus $18.3, \mathrm{p}=0.628$; IL-12: 3.3 versus $3.0, \mathrm{p}=0.542$, IL-13: 5.2 versus $3.6, p=0.003$, TNF- $\alpha: 2.9$ versus $2.8, \mathrm{p}=0.686$.

The reproducibility of the kit is given by the manufacturer. To show the reproducibility for the analyzed patients, the median RSD for the detected compounds was analyzed. The median RSD was $35 \%, 39 \%$ and $37 \%$ for glaucoma females, males and females+males, respectively. The median RSD for the control patients was $32 \%$, $49 \%$ and $45 \%$ for males, females and females+males, respectively.

We further evaluated the correlation between the levels of inflammatory cytokines and patients' clinical characteristics (IOP, number of glaucoma medications, topical use 


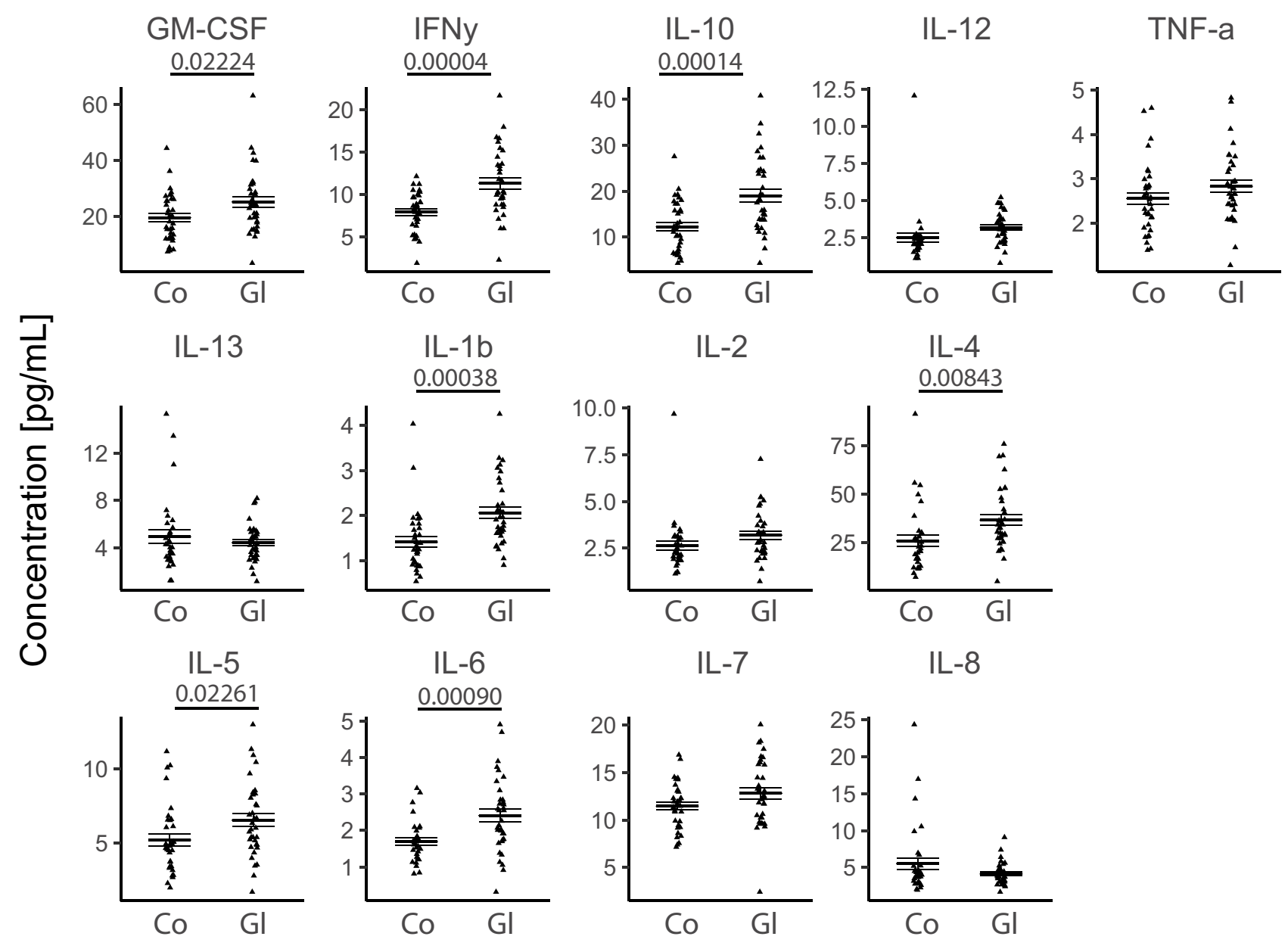

Figure 3 Sex-independent jitter plots of the concentration $(\mathrm{pg} / \mathrm{mL})$ for the measured analytes. Biological replicates are plotted individually. The overall mean $\pm S E M$ is overlaid.

Abbreviations: Co, control group; Gl, glaucoma group.

of prostaglandins, CDR, RNFL). GM-CSF and IL-10 were significantly correlated with IOP in POAG (Table 4). Additionally, IFN- $\gamma$, IL-1 $\beta$, IL-4, IL-5, IL-10 and IL-12 were significantly positively correlated with the use of topical prostaglandins. In our study, there was no significant correlation for CDR, glaucoma medication or RNFL measurements in the glaucoma cohort.

The backwards stepwise multiple regression analysis revealed no significant difference between the different variables age $(\beta=0.62 ; 95 \% \mathrm{CI}=-0.17-1.41 ; \mathrm{p}=0.12)$, $\operatorname{sex}(\beta=1.53 ; 95 \% \mathrm{CI}=-21.86-24.92 ; \mathrm{p}=0.90)$ and controlPOAG group $(\beta=-5.76 ; 95 \% \mathrm{CI}=-34.30-22.77 ; \mathrm{p}=0.70)$ and the NUCB2/nesfatin-1 (all $\mathrm{p}>0.05 ; \mathrm{R}^{2}=0.048$ ).

\section{Discussion}

\section{The Role of Nesfatin-I in POAG}

Neuroinflammatory mechanisms are a key factor in the pathogenesis of POAG. ${ }^{14-17}$ NUCB2/nesfatin-1 showed in animal models of testicular injury and cerebral trauma, that pretreatment significantly reduced the expression of pro-inflammatory cytokines and the apoptosis rate. ${ }^{12,33} \mathrm{We}$ assume that Nesfatin-1 might be a possible biomarker with neuroprotective properties pointing out the retinalhypothalamic axis as target site in POAG.

In our study, a slight but not significant increase in NUCB2/nesfatin-1 is prominent for male glaucoma patients compared to the control group. We did not find any significant sex-independent or sex-dependent differences in NUCB2/nesfatin-1 levels. Furthermore, no significant correlation between clinical glaucoma parameters such as CDR, RNFL and IOP, and NUCB2/nesfatin-1 was found. Thus, we did not find a clear context in plasma concentrations of NUCB2/nesfatin-1 and POAG (no correlation in multiple regression analysis).

In addition, our results showed a noticeable correlation of IL-13 and IL-2 with the Nesfatin-1 concentration in POAG. IL-13 was significantly downregulated in the male 


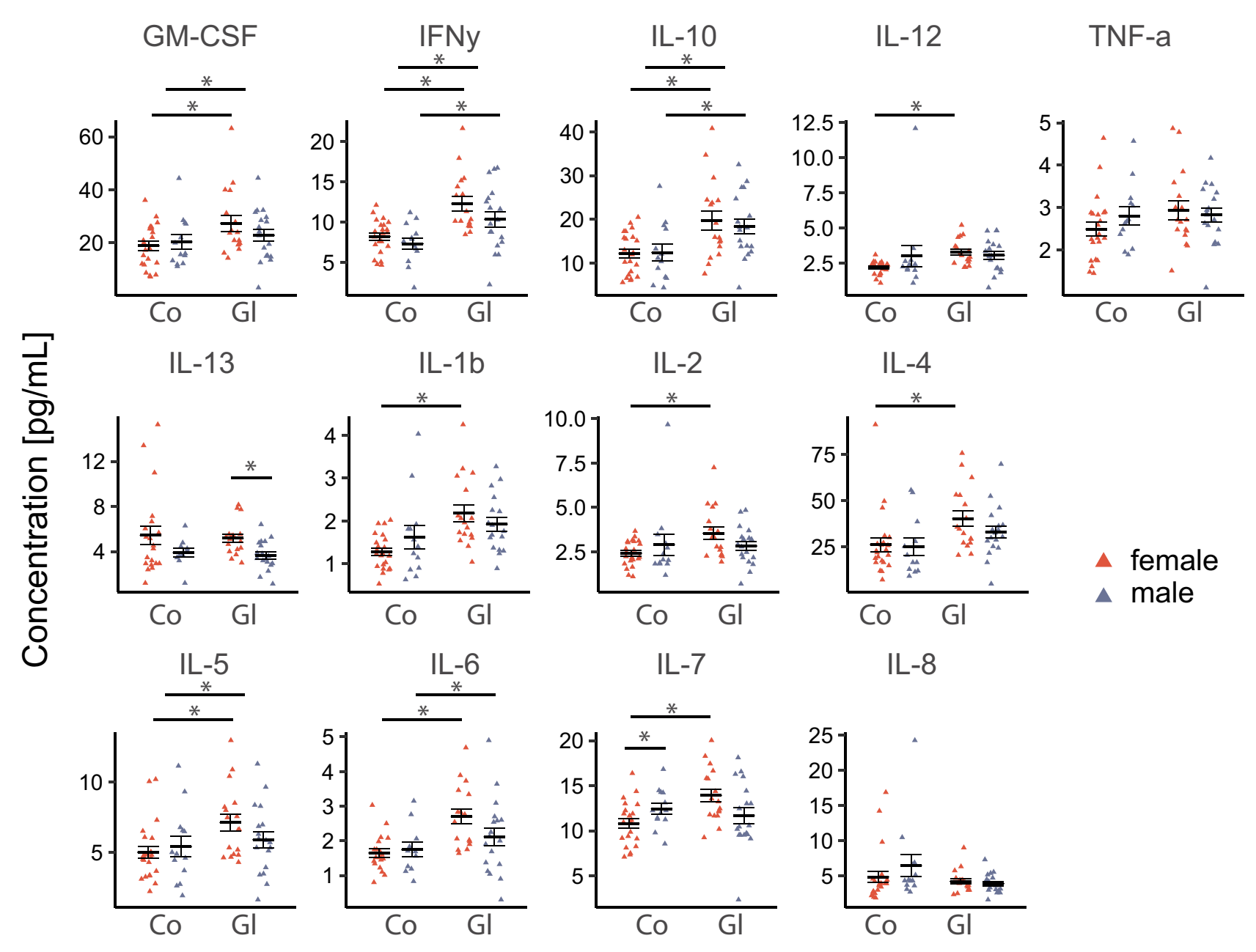

Figure 4 Sex-dependent jitter plots of the concentration $(\mathrm{pg} / \mathrm{mL})$ for the measured analytes. Biological replicates are plotted individually. The overall mean \pm SEM is overlaid. ${ }^{*} \mathrm{p}<0.05$. Blue $=$ male, red $=$ female.

Abbreviations: Co, control group; Gl, glaucoma group.

glaucoma cohort compared to female POAG. IL-2 was upregulated in glaucoma patients, especially in the female cohort. IL-2 plays an important role in the development of the T-cell immunologic memory and activates programmed cell death linked to FAS receptors. ${ }^{34}$ An upregulation might interact with the apoptosis of retinal ganglion cells in glaucoma. Furthermore, it is presumed that IL-13 activates matrix metalloproteinases (MMPs) to inhibit overexpression of allergic and inflammatory precursor molecules, which indicates its anti-inflammatory property. ${ }^{35}$

Referring to the cytokine status, there is a general lack of comparative research analysing NUCB2/nesfatin-1 levels in comparison to plasma cytokine concentrations. One study reported about cytokine concentrations in a depression model. ${ }^{36}$ In this study, a slight correlation of NUCB2/nesfatin-1 to the pro-inflammatory cytokine IL-6 $(r=0.333$, $\mathrm{p}=0.003)$ and $\operatorname{CRP}(\mathrm{r}=0.244, \mathrm{p}=0.034)$ was found. ${ }^{36}$
IL-2 plays an important role in the development of the T-cell immunologic memory and activates programmed cell death linked to FAS receptors. ${ }^{34}$ An upregulation might interact with the apoptosis of retinal ganglion cells in glaucoma.

Our study is limited by the lack of data on gonadal hormones. For future projects, subsequent studies following the trend of elevated NUCB2/nesfatin-1 levels in glaucoma male patients compared to healthy males could prove to be meaningful in escalating the study parameters and including gonadal hormones.

Additionally, NUCB2/nesfatin-1 concentrations might have even more informative value when using aqueous humour probes. Furthermore, we do not have an agematched control group, which might limit the explanatory power by assuming reduced testosterone levels with increasing age. 


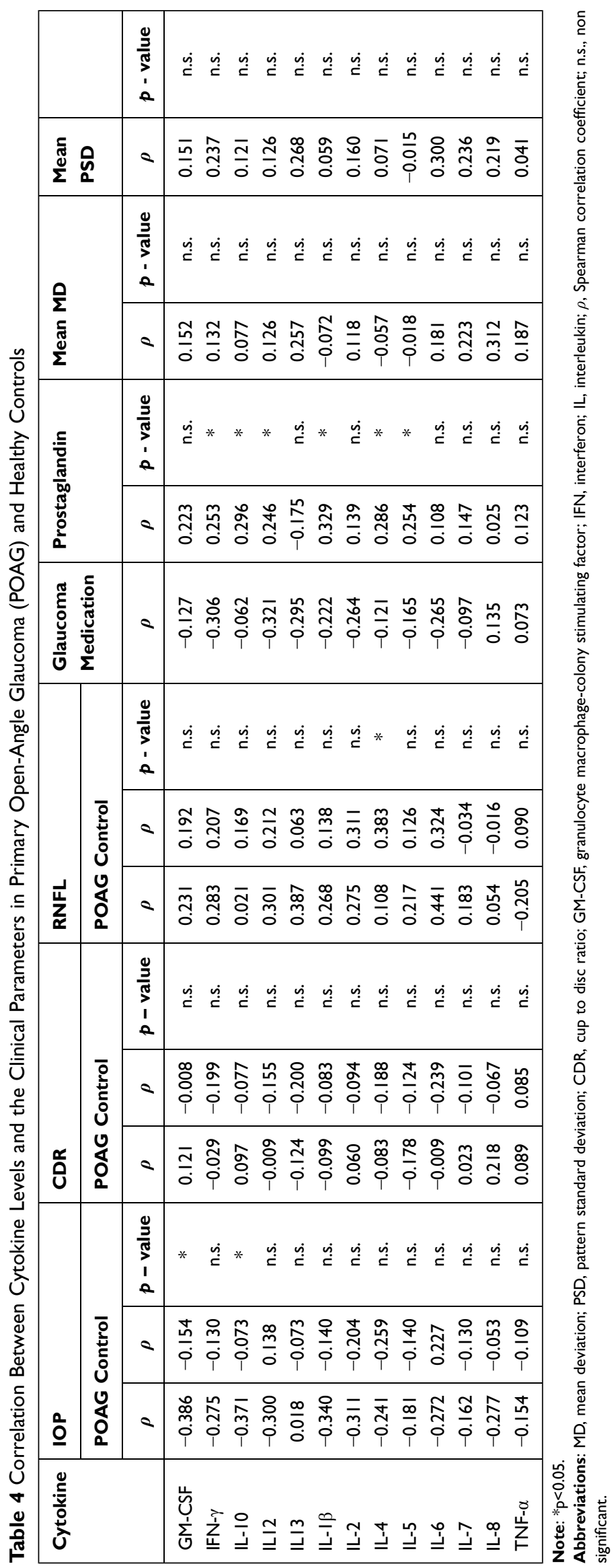




\section{Molecular Signature of POAG Patients}

Recent studies have provided evidence indicating a link between the IOP elevation and T cell-mediated autoimmune responses, which might underlie the pathogenesis of neurodegeneration in glaucoma. ${ }^{37}$ The proof that the adoptive transfer of $\mathrm{T}$ cells from glaucomatous mice results in a progressive loss of RGCs and their axons in recipient mice with a normal IOP supports the pathogenic role of $\mathrm{T}$ cells in glaucomatous neurodegeneration. ${ }^{37}$ However, the objective evaluation of $\mathrm{T}$ cells remains limited in patients and experimental models of glaucoma. There are only few studies focusing on the presence of specific $\mathrm{T}$ lymphocyte subsets in the sera of glaucoma patients. Thus, we decided to study the $\mathrm{T}$ cell specific response in the sera of our glaucoma patients, utilizing a premixed human high sensitivity $\mathrm{T}$ cell multiplex assay. The present study revealed significantly increased concentrations of 11 cytokines in glaucoma patients compared to the control group, except for IL-13 and IL-8, which showed decreased concentrations. This is in line with the literature, where an increasing body of evidence suggests neuroinflammation as a key process in glaucoma. ${ }^{14}$ In agreement with the literature on POAG patients, we found elevated levels of IFN-y, TNF- $\alpha$, IL-2, IL-4, IL-5, IL-6, IL-10 and IL-12. Our study adds valuable insights to the complex inflammatory process in POAG as we found elevated levels of GM-CSF, IL-1 $\beta$ and IL-7. Among all upregulated cytokines in our study, there were pro-inflammatory cytokines (IL-1 $\beta$, IL-6, IL-7, IL-12, GM-CSF, TNF- $\alpha$, IFN- $\gamma$ ) and anti-inflammatory cytokines (IL-10, IL-4, IL-5) and cytokines of neutrophil recruitment and activation (IL-1 $\beta$, TNF- $\alpha$ ). Various cellular sources were possible for the observed immune response. Interferon- $\gamma, \mathrm{TNF}-\alpha$, IL-2 and GM-CSF could be upregulated due to T-helper 1 cell (Th1) activation, whereas IL-4, IL-5, IL-6, IL-10 were secreted by Th2 cells. A possible source of IL-1 $\beta$, IL-6, TNF- $\alpha$ and GM-CSF are circulating monocytes or microglia, the resident macrophages of the eye.

The observed activation of immune cells (microglia in particular) and elevated levels of cytokines may be causative in RGC loss in glaucoma. Studies have shown that microglia increases in numbers and becomes activated in human and animal models of glaucoma. ${ }^{28,29,38,39}$ Additionally, there is some evidence that retinal and $\mathrm{ONH}$ microglia deactivation by minocycline at early stages of disease could result in reduced neurodegeneration. ${ }^{40,41}$ Furthermore, TNF- $\alpha$ produced by astrocytes and microglia has been demonstrated to promote RGC cell death, through a variety of mechanisms. ${ }^{42,43}$

We detected increased levels of IL- $1 \beta$, TNF- $\alpha$ and IL6 , maybe as attempt to prevent RGC cell loss and restore tissue homoeostasis. In the eye, for example, TNF- $\alpha$, IL$1 \alpha$ and IL-1 $\beta$ stimulated increased levels of MMP 3, MMP-9, and MMP-12, which are essential in maintaining IOP homeostasis by influencing extracellular matrix turnover in the trabecular meshwork - the outflow facility of the eye. Recent studies have also demonstrated that IL-6 acts as neuroprotectant and has the ability to increase the survival and regeneration of RGCs in vitro. Interleukin-6 mRNA and protein are upregulated near RGCs and their axons in rodent models of glaucoma. ${ }^{44,45}$

An important aspect of glaucomatous eyes is the presence of IOP elevation and fluctuation. Our results show that GM-CSF and IL-10 are significantly correlated with IOP in POAG. This is in line with corresponding literature which has found a positive correlation of patients' IOP measurements and elevated levels of cytokines. These studies concluded that IOP may be responsible for the production of cytokines. ${ }^{46,47}$

There are some limitations to this study. We detected the cytokines in the plasma, which might not be identical to the intraocular levels of these cytokines. In the literature, studies comparing levels of inflammatory cytokines in the peripheral blood and $\mathrm{AH}$ in various ocular diseases could, in part, show a similar cytokine profile in serum and intraocular fluid, especially for IL-1 $\beta$, IL-8, IL-10 and TNF- $\alpha .{ }^{48}$ Furthermore, in a young, healthy eye, the bloodretina barrier $(\mathrm{BRB})$ provides a tight regulation of molecular components crossing from the blood to the retina and vice versa. It is known that the BRB is impacted in human glaucomatous subjects and rodent models of glaucoma. ${ }^{49}$ Furthermore, the blood-brain barrier (BBB) around the $\mathrm{ONH}$ has been shown to leak in glaucomatous eyes. ${ }^{50}$ The probable relationship of impaired BRB and/or BBB to the pathogenesis of glaucoma suggests that inflammatory responses may spread into the circulation and thus could be detected in the peripheral blood as well. Another limitation is the fact, that patients with use of topical prostaglandins were included in the study. Six of the 13 measured cytokines were positively correlated with the topical prostaglandin use in our patient cohort. However, it has been demonstrated in the literature, that topical prostaglandins exhibit only a minor effect on the systemic bloodstream, especially in contrast to beta blocker medication. $^{51}$ 


\section{Conclusion}

In conclusion, we could detect a significant dependency in regard to the patients' sex of the cytokine concentration in POAG patients compared to the healthy population. A higher inflammatory response in POAG females compared to men was found. Additionally, NUCB2/nesfatin-1 plasma levels were slightly increased in the male glaucoma cohort. We conclude that these preliminary data are adding important information to the understanding of Nesfatin-1 actions in a glaucoma cohort. To the best of our knowledge, these valuable insights were not subject to comparative research prior to conducting this study.

\section{Abbreviations}

CDR, cup to disc ratio; GABA, gamma-aminobutyric acid; HRT, Heidelberg retina tomography; IL, interleukin; IOP, intraocular pressure; $\mathrm{MD}$, mean deviation; NTG, normal tension glaucoma; OAG, open-angle glaucoma; OCT, optical coherence tomography; PSD, pattern standard deviation; POAG, primary open-angle glaucoma; RGC, retinal ganglion cell; RNFL, retinal nerve fiber layer thickness.

\section{Author Contributions}

All authors contributed to data analysis, drafting or revising the article, have agreed on the journal to which the article will be submitted, gave final approval of the version to be published, and agree to be accountable for all aspects of the work. All authors made a significant contribution to the work reported, whether that is in the conception, study design, execution, acquisition of data, analysis and interpretation, or in all these areas; took part in drafting, revising or critically reviewing the article; gave final approval of the version to be published; have agreed on the journal to which the article has been submitted; and agree to be accountable for all aspects of the work.

\section{Funding}

We acknowledge Jennifer Kirwan from the Berlin Institute of Health (BIH) Metabolomics Platform for providing the MagPix Instrument and the possibility to perform the ELISA experiments. This research did not receive any specific grant from funding agencies in the public, commercial, or not-for-profit sectors.

\section{Disclosure}

Dominika Pohlmann is a participant in the BIH Charite Clinician Scientist Program funded by the Charite -
Universitätsmedizin Berlin and the Berlin Institute of Health. She reports travel and lecture fees from Allergan and Bayer. Anne Rübsam is a participant in the BIH Charite Clinician Scientist Program funded by the Charite - Universitätsmedizin Berlin and the Berlin Institute of Health. She reports a grant of the Deutsche Forschungsgemeinschaft (DFG) RU 2020/3-1. Carl Erb reports lecture fees from Allergan, Alcon, Bausch\&Lomb, Bayer, Novartis, Thea, Santen, Glaukos, Zeiss, Oculus, Visufarma. Consulting and advisory board fees from Allergan, OmniVision, Santen. Anna-Karina B Maier reports congress fees from Allergan and Novartis. Traveling expenses and lecture fees from Allergan. Sibylle Winterhalter has served as consultant for: Allergan, Novartis, Bayer, Heidelberg Engineering. Milena Pahlitzsch, Raphaela Fritsche-Guenther, and Inga Pompös report no financial disclosures. The authors report no other conflicts of interest in this work.

\section{References}

1. Pascolini D, Mariotti SP. Global estimates of visual impairment: 2010. Br J Ophthalmol. 2012;96(5):614-618. doi:10.1136/bjophthalmol-2011-300539

2. Nakazawa T, Nakazawa C, Matsubara A, et al. Tumor necrosis factor-alpha mediates oligodendrocyte death and delayed retinal ganglion cell loss in a mouse model of glaucoma. J Neurosci. 2006;26(49):12633-12641. doi:10.1523/JNEUROSCI.2801-06.2006

3. Tezel G, Li LY, Patil RV, Wax MB. TNF-alpha and TNF-alpha receptor-1 in the retina of normal and glaucomatous eyes. Invest Ophthalmol Vis Sci. 2001;42(8):1787-1794.

4. Vajaranant TS, Nayak S, Wilensky JT, Joslin CE. Gender and glaucoma: what we know and what we need to know. Curr Opin Ophthalmol. 2010;21(2):91-99. doi:10.1097/ICU.0b013e3283360b7e

5. Oh IS, Shimizu H, Satoh T, et al. Identification of nesfatin-1 as a satiety molecule in the hypothalamus. Nature. 2006;443 (7112):709-712. doi:10.1038/nature05162

6. Gong JL, Lou XT, Yuan YX, et al. The increased expression of GABA receptors within the arcuate nucleus is associated with high intraocular pressure. Mol Vis. 2018;24:574-586.

7. Ayada C, Toru U, Korkut Y. Nesfatin-1 and its effects on different systems. Hippokratia. 2015;19(1):4-10.

8. Pan W, Hsuchou H, Kastin AJ. Nesfatin-1 crosses the blood-brain barrier without saturation. Peptides. 2007;28(11):2223-2228. doi:10.1016/j.peptides.2007.09.005

9. Price TO, Samson WK, Niehoff ML, Banks WA. Permeability of the blood-brain barrier to a novel satiety molecule nesfatin-1. Peptides. 2007;28(12):2372-2381. doi:10.1016/j.peptides.2007.10.008

10. Samuels BC, Hammes NM, Johnson PL, Shekhar A, McKinnon SJ, Allingham RR. Dorsomedial/Perifornical hypothalamic stimulation increases intraocular pressure, intracranial pressure, and the translaminar pressure gradient. Invest Ophthalmol Vis Sci. 2012;53 (11):7328-7335. doi:10.1167/iovs.12-10632

11. Guzman-Ruiz M, Saderi N, Cazarez-Marquez F, et al. The suprachiasmatic nucleus changes the daily activity of the arcuate nucleus alpha-MSH neurons in male rats. Endocrinology. 2014;155 (2):525-535. doi:10.1210/en.2013-1604 
12. Ozsavci D, Ersahin M, Sener A, et al. The novel function of nesfatin-1 as an anti-inflammatory and antiapoptotic peptide in subarachnoid hemorrhage-induced oxidative brain damage in rats. Neurosurgery. 2011;68(6):1699-1708; discussion 1708. doi:10.1227/ NEU.0b013e318210f258

13. Davis BM, Tian K, Pahlitzsch M, et al. Topical Coenzyme Q10 demonstrates mitochondrial-mediated neuroprotection in a rodent model of ocular hypertension. Mitochondrion. 2017;36:114-123. doi:10.1016/j.mito.2017.05.010

14. Chen SD, Wang L, Zhang XL. Neuroprotection in glaucoma: present and future. Chin Med J (Engl). 2013;126(8):1567-1577.

15. Tian K, Shibata-Germanos S, Pahlitzsch M, Cordeiro MF. Current perspective of neuroprotection and glaucoma. Clin Ophthalmol. 2015;9:2109-2118. doi:10.2147/OPTH.S80445

16. Soto I, Howell GR. The complex role of neuroinflammation in glaucoma. Cold Spring Harb Perspect Med. 2014;4(8):a017269a017269. doi:10.1101/cshperspect.a017269

17. Xu H, Chen M, Forrester JV. Para-inflammation in the aging retina. Prog Retin Eye Res. 2009;28(5):348-368. doi:10.1016/j. preteyeres.2009.06.001

18. Zhou X, Li F, Ge J, et al. Retinal ganglion cell protection by 17-betaestradiol in a mouse model of inherited glaucoma. Dev Neurobiol. 2007;67(5):603-616. doi:10.1002/dneu.20373

19. Huang P, Qi Y, Xu YS, et al. Serum cytokine alteration is associated with optic neuropathy in human primary open angle glaucoma. J Glaucoma. 2010;19(5):324-330. doi:10.1097/IJG.0b013e3181b4cac7

20. Huang $\mathrm{P}$, Zhang SS, Zhang C. The two sides of cytokine signaling and glaucomatous optic neuropathy. J Ocul Biol Dis Infor. 2009;2 (2):78-83. doi:10.1007/s12177-009-9026-6

21. Huang W, Chen S, Gao X, et al. Inflammation-related cytokines of aqueous humor in acute primary angle-closure eyes. Invest Ophthalmol Vis Sci. 2014;55(2):1088-1094. doi:10.1167/iovs.13-13591

22. Wang Y, Chen S, Liu Y, Huang W, Li X, Zhang X. Inflammatory cytokine profiles in eyes with primary angle-closure glaucoma. Biosci Rep. 2018;38(6). doi:10.1042/BSR20181411

23. Wang WH, McNatt LG, Pang IH, et al. Increased expression of serum amyloid A in glaucoma and its effect on intraocular pressure. Invest Ophthalmol Vis Sci. 2008;49(5):1916-1923. doi:10.1167/iovs.07-1104

24. Chono I, Miyazaki D, Miyake H, et al. High interleukin-8 level in aqueous humor is associated with poor prognosis in eyes with open angle glaucoma and neovascular glaucoma. Sci Rep. 2018;8 (1):14533. doi:10.1038/s41598-018-32725-3

25. Takano Y, Shi D, Shimizu A, et al. Association of Toll-like receptor 4 gene polymorphisms in Japanese subjects with primary open-angle, normal-tension, and exfoliation glaucoma. Am J Ophthalmol. 2012;154(5):825-832 e821. doi:10.1016/j.ajo.2012.03.050

26. Wang CY, Shen YC, Wei LC, et al. Polymorphism in the TNF-alpha (-863) locus associated with reduced risk of primary open angle glaucoma. Mol Vis. 2012;18:779-785.

27. Wei X, Cho KS, Thee EF, Jager MJ, Chen DF. Neuroinflammation and microglia in glaucoma: time for a paradigm shift. J Neurosci Res. 2019;97(1):70-76. doi:10.1002/jnr.24256

28. Howell GR, Macalinao DG, Sousa GL, et al. Molecular clustering identifies complement and endothelin induction as early events in a mouse model of glaucoma. J Clin Invest. 2011;121(4):1429-1444. doi:10.1172/JCI44646

29. Bosco A, Steele MR, Vetter ML. Early microglia activation in a mouse model of chronic glaucoma. J Comp Neurol. 2011;519 (4):599-620. doi:10.1002/cne.22516

30. Bosco A, Inman DM, Steele MR, et al. Reduced retina microglial activation and improved optic nerve integrity with minocycline treatment in the DBA/2J mouse model of glaucoma. Invest Ophthalmol Vis Sci. 2008;49(4):1437-1446. doi:10.1167/iovs.07-1337
31. Howell GR, Soto I, Zhu X, et al. Radiation treatment inhibits monocyte entry into the optic nerve head and prevents neuronal damage in a mouse model of glaucoma. J Clin Invest. 2012;122(4):1246-1261. doi: $10.1172 /$ JCI61135

32. Naganuma F, Bandaru SS, Absi G, Chee MJ, Vetrivelan R. Melaninconcentrating hormone neurons promote rapid eye movement sleep independent of glutamate release. Brain Struct Funct. 2019;224 (1):99-110. doi:10.1007/s00429-018-1766-2

33. Arabaci Tamer S, Yildirim A, Koroglu MK, Cevik O, Ercan F, Yegen BC. Nesfatin-1 ameliorates testicular injury and supports gonadal function in rats induced with testis torsion. Peptides. 2018;107:1-9. doi:10.1016/j.peptides.2018.07.005

34. Arenas-Ramirez N, Woytschak J, Boyman O. Interleukin-2: biology, design and application. Trends Immunol. 2015;36(12):763-777. doi:10.1016/j.it.2015.10.003

35. Minty A, Chalon P, Derocq JM, et al. Interleukin-13 is a new human lymphokine regulating inflammatory and immune responses. Nature. 1993;362(6417):248-250. doi:10.1038/362248a0

36. Xia QR, Liang J, Cao Y, Shan F, Liu Y, Xu YY. Increased plasma nesfatin-1 levels may be associated with corticosterone, IL-6, and CRP levels in patients with major depressive disorder. Clin Chim Acta. 2018;480:107-111. doi:10.1016/j.cca.2018.02.004

37. Gramlich OW, Ding QJ, Zhu W, Cook A, Anderson MG, Kuehn MH. Adoptive transfer of immune cells from glaucomatous mice provokes retinal ganglion cell loss in recipients. Acta Neuropathol Commun. 2015;3:56. doi:10.1186/s40478-015-0234-y

38. Howell GR, MacNicoll KH, Braine CE, et al. Combinatorial targeting of early pathways profoundly inhibits neurodegeneration in a mouse model of glaucoma. Neurobiol Dis. 2014;71:44-52. doi:10.1016/j.nbd.2014.07.016

39. Qu J, Jakobs TC, Di Giovanni S. The time course of gene expression during reactive gliosis in the optic nerve. PLoS One. 2013;8(6): e67094. doi:10.1371/journal.pone.0067094

40. Levkovitch-Verbin H, Waserzoog Y, Vander S, Makarovsky D, Ilia P. Minocycline mechanism of neuroprotection involves the Bcl-2 gene family in optic nerve transection. Int $J$ Neurosci. 2014;124 (10):755-761. doi:10.3109/00207454.2013.878340

41. Levkovitch-Verbin H, Waserzoog Y, Vander S, Makarovsky D, Piven I. Minocycline upregulates pro-survival genes and downregulates pro-apoptotic genes in experimental glaucoma. Graefes Arch Clin Exp Ophthalmol. 2014;252(5):761-772. doi:10.1007/s00417-014-2588-4

42. Mac Nair CE, Fernandes KA, Schlamp CL, Libby RT, Nickells RW. Tumor necrosis factor alpha has an early protective effect on retinal ganglion cells after optic nerve crush. $J$ Neuroinflammation. 2014;11:194. doi:10.1186/s12974-014-0194-3

43. Cueva Vargas JL, Osswald IK, Unsain N, et al. Soluble tumor necrosis factor alpha promotes retinal ganglion cell death in glaucoma via calcium-permeable AMPA receptor activation. $J$ Neurosci. 2015;35(35):12088-12102. doi:10.1523/JNEUROSCI.1273-15.2015

44. Sims SM, Holmgren L, Cathcart HM, Sappington RM. Spatial regulation of interleukin-6 signaling in response to neurodegenerative stressors in the retina. Am J Neurodegener Dis. 2012;1(2):168-179.

45. Sappington RM, Chan M, Calkins DJ. Interleukin-6 protects retinal ganglion cells from pressure-induced death. Invest Ophthalmol Vis Sci. 2006;47(7):2932-2942. doi:10.1167/iovs.05-1407

46. Tong Y, Zhou YL, Zheng Y, Biswal M, Zhao PQ, Wang ZY. Analyzing cytokines as biomarkers to evaluate severity of glaucoma. Int J Ophthalmol. 2017;10(6):925-930. doi:10.18240/ ijo.2017.06.15

47. Garweg JG, Zandi S, Pfister IB, Skowronska M, Gerhardt C. Comparison of cytokine profiles in the aqueous humor of eyes with pseudoexfoliation syndrome and glaucoma. PLoS One. 2017;12(8): e0182571. doi:10.1371/journal.pone.0182571 
48. Ten Berge JC, Fazil Z, van den Born I, et al. Intraocular cytokine profile and autoimmune reactions in retinitis pigmentosa, age-related macular degeneration, glaucoma and cataract. Acta Ophthalmol. 2019;97(2):185-192. doi:10.1111/aos.13899

49. Schwartz B. Circulatory defects of the optic disk and retina in ocular hypertension and high pressure open-angle glaucoma. Surv Ophthalmol. 1994;38(Suppl):S23-34. doi:10.1016/0039-6257(94) 90044-2
50. Arend O, Remky A, Plange N, Kaup M, Schwartz B. Fluorescein leakage of the optic disc in glaucomatous optic neuropathy. Graefes Arch Clin Exp Ophthalmol. 2005;243(7):659-664. doi:10.1007/ s00417-004-1092-7

51. Easthope SE, Perry CM. Topical bimatoprost: a review of its use in open-angle glaucoma and ocular hypertension. Drugs Aging. 2002;19 (3):231-248. doi:10.2165/00002512-200219030-00008

\section{Publish your work in this journal}

Clinical Ophthalmology is an international, peer-reviewed journal covering all subspecialties within ophthalmology. Key topics include: Optometry; Visual science; Pharmacology and drug therapy in eye diseases; Basic Sciences; Primary and Secondary eye care; Patient Safety and Quality of Care Improvements. This journal is indexed on PubMed

Submit your manuscript here: https://www.dovepress.com/clinical-ophthalmology-journal
Central and CAS, and is the official journal of The Society of Clinical Ophthalmology (SCO). The manuscript management system is completely online and includes a very quick and fair peer-review system, which is all easy to use. Visit http://www.dovepress.com/ testimonials.php to read real quotes from published authors. 